\title{
EST Translation Guided by Reception Theory ${ }^{*}$
}

\author{
Liangqiu Lv, Puyu Ning \\ School of Foreign Language, North China Electric Power University, Beijing, China \\ Email: 1lq2803@163.com \\ Received February $8^{\text {th }}$, 2013; revised March $10^{\text {th }}$, 2013; accepted March $18^{\text {th }}, 2013$
}

\begin{abstract}
Copyright (c) 2013 Liangqiu Lv, Puyu Ning. This is an open access article distributed under the Creative Commons Attribution License, which permits unrestricted use, distribution, and reproduction in any medium, provided the original work is properly cited.
\end{abstract}

\begin{abstract}
In the thesis, Reception Theory is introduced to the studies of EST translation to explore the mutual influence and restriction between the author, the translator and readers, as well as the respective reception problems, on the basis of which, the characteristics, principles and strategies of translation are analyzed.
\end{abstract}

Keywords: Reception Theory; EST; Translation Strategies

\section{Reception Theory and Translation Studies}

The focus on Reception Theory in the western academia was not originated from education but from the Hermeneutics of ancient Greece as well as the aesthetics of reception developed therefrom. Stemmed from Germany in the 70s of the 20th century, Reception Theory, or the aesthetics of reception, is one of the important literary theory schools. The main founders of the theory are Robert Jauss and Wolfgang Iser, both of whom are representatives of the Constance school. The two research orientations-reception research and effect research, interrelated and different from each other, have been formed in the development of Reception Theory.

Represented by Robert Jauss, reception research systematically expounds the basic theory of the aesthetics of reception. He focused on readership study and concerned with the horizon of expectation and the aesthetic experience of readers. He thought that the reception process is a positive, not negative participation process. Since the social circumstance of each historical period forms certain standards and paradigms, while the talents, experience and cultural cultivation of each recipient are different, therefore the understanding and comprehension towards works by different recipients vary in quite wide range. For a certain piece of work, it will present various meaning structure under different background of history and society; and under the same social and historical background, it will also have different meaning structure to different readers.

With the interaction between text and reader as well as the viewpoint which the meaning developed in the reading process as the logical starting point, effect research represented by Wolfgang Iser stresses the text study during the reception and focuses on the blank and appealing structure of text. He believed that meaning is not the product of a single aspect of text or reader. Before being received by reader, the text is just an unascertained "appealing structure" awaiting realization. Only through the proactive exploration by reader during the reception

*The present paper is one of the periodical results of the Project "the Application of Empathy and Cultural Perception in Translation Process” (No. 12MS91), supported by the Fundamental Research Funds for the Central Universities of China. can the meaning of text be realized. During the reading and receiving course, the thinking process of reader is very vital.

It is reception theory that first put forward from the height of ontology the issue of reader and reading reception always ignored by people. The starting points of the Construction Reception Theory of Rober Jauss and Iser are consistent. The focuses of both theories are diverted from the relation from author to text, to the study of the relation from text to author. And the important role of reader in the whole process of literary activity is served as the basis of both theories (Zhou \& Jin, 1987).

In late 80s and early 90s of the 20th century, Reception Theory was introduced to translation study. Translation researchers started to turn their focus from translations and translators to the acceptance of translations by readers. The status and role of readers in the translation process as well as influences of reader acceptance on the translation goals, strategies, etc. were highlighted.

There are two acceptance activities in translation practice. The reading of the source text by translator is the first acceptance during which he will fill up with creativity a lot of undetermined blanks in the source text. Robert Jauss believed that the significance of works originates both from the works itself and from the readers. No translator can be excluded from the influence of his reading experience and prior knowledge. Meanwhile, the source text restricts the acceptance of reader. So the reader will not deviate from the meaning of the source text or understand randomly, and have the translation approximate to the source text as much as possible. While seeking the utmost approximation between the translation and the source text, the translator should also consider the relation as well as the fusion of horizon between translation and its reader. Undoubtedly, the communication between the readers of translation will also influence the translation activity of the translator, who needs to think about the acceptance ability and requirement of the readers in the translation and constantly have the translation communicate and dialogue with default readers. Meanwhile, the translator is also the reader of the source text and keeps communicating with the translation works as reader. The translator needs to precast the concurrent horizon of expectation of readers, take the concurrent aesthetic taste and acceptance ability of 
readers into consideration, and have the horizon of expectation of readers fused into his translation works.

\section{Characteristics and Translation Principles of EST}

\section{Characteristics of EST}

Generally, there are three characteristics for EST, namely, accuracy, objectivity and brevity, which are specifically embodied in the aspects of wording, sentence pattern and presentation: Use of large number of very professional words accompanied with lots of formulas, data, graphs and etc; use of long sentence and comparatively complicated sentence; impersonal; formal in mode of speech; objective and accurate in statement; standard in language; unadorned in stylistics; strict in logic and concentrated in technical terms (Wang \& Pan, 2009).

\section{Translation Principles for EST}

There are three basic principles in translation: psychological principle, message transferring principle, and aesthetic principle. The aesthetics principle denotes that translator needs to transfer the beauty in sense, sound and form of the original works in translation. (Liu Kezhang, 1994). In Sci-tech translation, the message transferring principle tops the list, which means that Sci-tech translator also needs to consider the acceptance of reading psychology of readers.

EST mainly states affairs, describes phenomenon, deduces formula, demonstrates rules, and includes less emotional colors. Therefore, principles of faithfulness, expressiveness, conciseness, accuracy and serving readers shall be followed in translation, so as to make the translation works clear and concise with well-knit structure, seamless logic, standard writing, and correct technical terms.

\section{Principle of Faithfulness}

All languages contain polysemic words, which is an inevitable result of language development. The meaning of specific words must be determined according to the specific linguistic context and logical relationship. Different style has different requirement on faithfulness. In sci-tech translation, faithfulness means that the meaning of the source text can be represented in an entirely accurate way in the target text. Meanwhile, the faithfulness of the style of the target text should also be valued. This is the basic requirement for sci-tech translation.

1) Faithful to the meaning of the source context

To be faithful to the content of the source text in translation, a translator should well grasp the general idea of the entire article on a macro basis first. Then, based on the basic idea of the source language and integrated with the context relation, logical relation and the institutionalized Chinese expression, he should select the appropriate word meaning conforming to scientific writing in translation, hence realizing the faithfulness of translation.

2) Faithful to the style of the source text

In EST translation, to faithfully convey the style of the source text is also very important. EST requires concise writing and well-knit structure, therefore, more nominalizations or participial phrases are used to replace attributive clause or adverbial clause. When translating such article, translator should try to keep his target text the same style with that of the source one.

\section{Principle of Expressiveness}

The main aim of EST translation is to transmit new knowledge in the field of science and technology, namely, the new theories, information and data unknown to readers before. Therefore, the target text should be as accurate, concise and standard as possible and embody its own characteristics of understanding and expression in translation. The target text should be in accordance with Chinese standard and be readable. In expression, translator should try his best to get rid of the structural shackles of the source text, pay attention to collocation as well as selection and extension of word meaning, and make the target text with smooth expression.

To follow the principle of expressiveness, translator needs to accurately translate the pure science and technology terms according to the background of the source text, to correctly understand and use the science and technology terms as well as the meaning of the words expressing abstract concept, and understand the derivates and abbreviations according to the general idea of the article. The Anglo-Americans overemphasize changes of sentence form, structure and vocabulary in writing, while the Chinese often use the same word to express the object, action or status with the same concept in sci-tech articles, so as to realize the accuracy in expression. If ignoring the differences of expression between the sci-tech articles of English and Chinese, translator will confuse concepts and his translation will not be smooth.

\section{Principle of Brevity}

The aim of sci-tech translation is to convey scientific knowledge to readers, which requires the adopted linguistic form, especially those scientific and technical terms to be concise, so that readers can correctly understand and grasp the contents. To make the translation concise, translator should fully understand the source text first, and delete the redundant modifications, conjunctions, prepositions and etc. therein on the precondition of not affecting the original contents.

\section{Principle of Accuracy}

Principle of accuracy refers to using target language to appropriately reflect the meaning of the source language in translation. The wording of EST must be correct without confusion or words with multiple meanings. Therefore, in sci-tech translation, translator should appropriately use the specific sentence structure, tense, voice, terms and etc of science and technology, and carefully analyze, differentiate and select the meaning of words on the basis of relevant specialized knowledge, so as to improve the accuracy of contents and simplicity of form.

\section{Principle of Serving Readers}

The aim of translation is to have reader clearly understand the information of the source text through reading the target text. Translation is to serve the readers of the target text. The response of the reader of the target text should be consistent to that of the reader of the source text as much as possible. Therefore, the target text should be concise, clear, smooth, standard, and be in compliance with the standard of target language. Translator should give full consideration of the understanding, capacity and perception of language of the reader of the target text, and properly simplify information.

Due to the difference of expression between English and Chinese, some words might have the same connotations but 
different extensions for the readers of the two languages. Besides, some words can be tactfully arranged in a certain phrase in native English; however, it is very difficult to find relevant collocation in Chinese. Only when translator has seized the essential characteristics and expressed appropriately, can he work out the real charm of the source text.

\section{EST Translation Guided by Reception Theory}

As a bilingual conversion activity, translation actually involves the original author, the translator and the readers of the target text, who form a tripartite confrontation situation with the three parties therein cooperative and restricted with each other, and contain and compromise with each other. The original author restricts the translator not to deviate from the source text. The translator is both the reader of the source text and the author of the target text. And he has to consider the acceptance and need of readers, so his translation can gain the expected result. Readers restrict the realization of translation. Meanwhile, the three parties need to cooperate with each other too. The original author should allow the translator to betray if necessary, and the readers cannot require the translator to consider their feeling only regardless of the source text. The translator should take both the original author and the readers into consideration, not only make his translation understandable, but also keep the style of the source text (Sun \& Zhao, 1998).

\section{Reception in EST Translation}

In EST translation, there is also reception which emphasizes the reception psychology and expectation towards the science and technology information in the source text, but not the feeling of beauty. The author, the translator and the readers will have either direct or indirect influence on reception. The style of the source text determines its target readership, hence the readership of the translator. Linking between the author and the readers, the translator should correctly convey the information of the source text, meet the need of the readers and serve the readers. This is bound to affect the selection and use of the translation strategies of the translator.

\section{Reception of the Author}

The reception of the author of the source text is reflected by the relation between style and relation. Meanwhile, the information can also reflect its relation with reception. Generally, the author of the source text will take the need and interest of readers into consideration in his writing. Although the difference of reception psychology and decoding ability between the readers of the source text and the readers of the target text is not big, their interests and need for the article may not be exactly the same, which causes the reception of the readers of the target text towards the information to be different from those of the source text. In addition, the readers' demands for information are not same either. Whether the value of the source text can be realized is determined by the reading demand of the readers of the target language. The source text is an integration of multilevel, multi-factor, and multi-category information. After being translated, the target text is faced with various types of readers. Some information is needed by readers while the others not. All of them will affect the reception of the translation. Both the style and information of the source text will have indirect influence on the reception of the readers of the target text. Ignor- ing these factors will affect the outcome and the realization of the value of the translation, worse still, the reception of the target text of the readers will also decrease. It is the translator's responsibility for readers to adjust the information in the source text and select the appropriate translation strategies according to the reception of readers.

\section{Reception of the Translator}

According to reception theory, the translator should not only pay attention to the faithful conveying of various information of the source text but also consider the reception ability of the readers of the translation. Placed between the author and the readers, the translator should get himself involved into both the creation activity of the author and the reading process of readers. He should know both the author and the readers, and should be responsible both for the original work and the readers (Fang Mengzhi, 2008).

Only those translations accepted by readers are excellent works. In sci-tech translation, the task of the translator is to faithfully and correctly convey the scientific and technical information. The translator's responsibility for reader lies in both faithfully and correctly conveying the information of the source text as well as considering the requirement and reception psychology of readers. Faithfulness and accuracy are the basic requirements of sci-tech translation as well as the foundation for realizing the goal of translation. Since sci-tech translation involves the specific contents in the field of science and technology, the translator must grasp the knowledge of the relevant disciplines in the source text and skillfully master language. On the basis of understanding the source text, he can use standard language to express the meaning in the target language in a correct, smooth and native way. Otherwise, the translator may make mistakes and lose the value of translation, let alone realize the aim of translation and the expected social result.

On the basis of faithfully conveying the information of the source text, the translator also needs to consider the demand of readers. Otherwise, his translation will affect the reception of readers. The translator must make clear of the aim of translating the article, sort out the information in the source text, and convey the contents badly needed by readers in his translation. For the translator, the demand of readers is a drive that can motivate his potentiality used in his translation.

\section{Reception of the Reader}

As previously mentioned, the principle of serving readers should be followed in sci-tech translation. The translator must consider the reception of readers. In sci-tech translation, the reception of readers refers to the reception of the information in the source text, which is where the value of the original text is realized. Like the other translations, only through the reading, thinking, and absorption of readers can the sci-tech translations influence society.

The reception of readers mainly includes reader psychology, reader structure, the characteristics of reader and etc. Reader psychology includes interest and attention. To attract readers and their attention, the translation must have something that can interest readers. If the translator can pay attention to reader psychology, and accordingly adjust the structure, the distribution of information and selection of information point it in his translation, the quality of his translation can be improved (Fang, 1992). 
In terms of reader structure, there are professionals and nonprofessionals. For the professional technology personnel, their point of interest lies in their profession. All scientific achievements related to their professional research can attract their attention. What they are mostly concerned and interested is the development prospect of their profession. The translator had better start from their point of interest when selecting the source text, then the target text can attract the attention of the professional technology personnel, who have strong comprehension, so the translation should be concise and clear. A large number of specialized, simplified or conventionalized terms can be directly used. The translator cannot make his translation shallow and vulgarized.

For non-professional readers, their interests mainly lie in new knowledge or the information about new technology. They are not interested in the new technology in a certain field. So to speak, their interests are the widest but most unsteady ones. Their gaining of certain scientific knowledge is not always intentional or on purpose. For such readers, the title of the translation is very important. It should be able to not only highlight the key information but also attract the reading interests of readers. Besides, such translations should adjust the distribution as well as the primary and secondary of information in the source text according to the interest of readers. The information interested by readers or useful to the living, working and studying of readers should be placed in front. If the translator only speculates the reception psychology of the readers of the target text according to the information reception of the readers of the source text, his translation works will not exert its due influence.

In addition, the translator should also know the reception ability of readers, so as to avoid the misplaced reception. The translator must start from the reception ability, interests and requirement of the readers of his translation, revise the source text, and convey the information in the source text to readers in the way that they are willing to accept.

\section{Strategies of EST Translation Guided by Reception Theory}

Apart from the selection of the information in the source text and the wording and phrasing of the target text, the translator of EST must have reader awareness on the selection and use of translation strategies. He needs to consider about the reception of readers. On the basis of correctly conveying the information of the source text, he should try to make his translation with the best readability.

\section{Foreignization of EST Translation}

It should become the primary responsibility of the translators of EST to correctly convey the information of the source text and set faithfulness as the highest principle of sci-tech translation In EST translation, the translator often locks some special language structures, reserves the language characteristics of the source text and follows the fixed expression. Although sometimes this kind of expression sounds awkward with strong foreign flavor, it is a kind of inevitable foreignization, which is just a reflection of thinking of the reception of the author. The language structures needing to be locked by the translator in his translation mainly are some technical terms which have fixed translation. The translator cannot translate them at his will but look up relative technical dictionary and follow suit.
Apart from some single special terms, the language structures to be locked in sci-tech translation also include some larger language units such as phrases, which have formed fixed concept in some special fields and have conventionalized translation. In terms of the phrase-form special terms, the translator should not translate their fixed expressions at will but pay attention to them in this field and consider the reception of readers.

\section{Domestication of EST Translation}

Translation is not only a conversion between two languages; it also involves clashes and restrictions between two cultures. EST stresses the professional expression of terms, the direct, objective and logical presentation, and the invariance of style. When translating the source text to the target text, the translator needs to instill his re-creational elements; therefore, the translation will inevitably be marked with the culture of the target language. The translator has his own free processing and creating space in translation. During the translation, he should consider about the reception of readers. Only when the translator gets familiar with the readers of the target language, realizes the reception of readers and makes his translation standard, smooth and fluent with the cultural elements of the target language, can his works be successful.

Undoubtedly, the terms and phrases should be faithfully translated in sci-tech translation. But it does not mean that the characteristics belonging to language system and without any professional meaning should be unreservedly inherited in translation. As is well known, one of the most outstanding characteristics in EST is nominalization. Something expressed with verbs in daily communication is often expressed with nouns in text, for nominal language structure can often avoid the changes of tense, hence making the language more objective. However, such nominal language structure does not bear too much language information. It is just a language tool forming the discourse of science and technology. Therefore, when translating such nominal structures, the translator has to use verb structure to replace the nominal structure in the source text according to the Chinese way of expression of using verbs more, to ensure the clear and coherent of the target text.

There are comparatively big cultural differences between Chinese readers and English readers. Some background knowledge of science and technology English readers are familiar with may be unknown to most Chinese readers. In this case, it is necessary for the translator to appropriately supplement background knowledge and expand the co-occurred information of the source text, or provide the information the western readers are familiar with but the Chinese readers are not, so as to cross the cultural background barrier and help readers to better understand the meaning of the source text. For readers, there are some scientific barrier in ECST (English for Common Science and Technology) itself. Too many foreign expressions will affect the understanding of readers and reduce their reading interests.

\section{Conclusion}

Since there is reception in sci-tech translation, the translator should consider the feeling and demand of the readers in his translation so as to have his translation meet the demand of readers. The sci-tech translation emphasizes the reception of the science and technology information contained in the source text 
by the readers of the target text, but not the same feeling as that of the readers of the source text by the readers of the target text. Therefore, the sci-tech translation stresses that the translator should translate according to the demand and need of the readers, which is actually an intensification and utmost use of the reception aesthetics. Since the readers affect the translation so seriously, it is necessary for the translator to select appropriate strategies according to the acceptance, understanding and need of the readers to translate. The source text, readers and translators will affect reception. The style of the source text determines its target readership, hence the target readership of the target text. Then the translator also has close relations with reception. Situated between the author and the readers, the translator should use various methods to meet the readers' demand for the source text, adjust the structure, or make selections of the information of the source text and etc. The reception ability and reading interests of the readers themselves will also affect the acceptance of the information in target text.

\section{REFERENCES}

Zhou, N., \& Jin, Y. P. (1987). Reception aesthetics and reception theory. Shenyang: Liaoning People's Publishing House.

Fang, M. Z., \& Fan, W. Q. (2008). A Course in sci-tech translation. Shanghai: Shanghai Foreign Language Education Press.

Wang, W. P., Pan, L. R., et al. (2009). Linguistic features \& translation. Shanghai: Shanghai Jiao Tong University Press.

Fang, M. Z. (1992). How does translation be accepted by readers? Shanghai Journal of Translators for Science and Technology.

Sun, H. J., \& Zhao, X. J. (1998). Relations between the original author, the translator and the readers of the target text in translation. Chinese Translators Journal.

Liu, K. Z. (1994). The basic principles in translation. Beijing: China Science and Technology Press.

Chen, L. L. (1999). The foreignization and domestication of translation. Chinese Science \& Technology Translators Journal. 